\title{
Le nouveau lycée : une occasion manquée...
}

Le 31 janvier dernier, Luc Chatel, ministre de l'Éducation nationale, de la jeunesse et de la vie associative, présentait devant la presse les mesures d'une nouvelle ambition pour les sciences et les technologies à l'École. Entre autres mesures y étaient détaillées celles visant à développer le goût de ces disciplines au collège, à décloisonner leur approche pour redonner du sens à l'enseignement, ou encore à encourager les vocations pour les carrières scientifiques et technologiques au lycée.

L'annonce de ce plan faisait suite à la publication, le 7 décembre 2010, du classement PISA 2009 de I'OCDE, où la France se classe au peu glorieux $27^{\mathrm{e}}$ rang sur 65 pour la culture scientifique. Il est intéressant de se plonger dans les constatations du rapport PISA. Nous en relevons seulement certaines ici : Les systèmes d'éducation les plus performants, ceux des territoires chinois de Shanghai et de Hong-Kong, de la Finlande et de Singapour, dispensent un enseignement de qualité à tous les élèves. Ils assurent - en outre - l'égalité des chances à tous, quel que soit le milieu socio-économique d'origine, tout en accordant une plus grande autonomie aux établissements dans l'élaboration des programmes et des politiques d'évaluation, sans toutefois permettre nécessairement la concurrence entre établissements. À l'échelle de l'élève, le milieu familial influe sur la réussite scolaire, et l'école semble souvent en renforcer l'impact. Vivre dans un milieu socio-économique défavorisé n'entraîne cependant pas nécessairement de mauvais résultats scolaires, mais le profil socio-économique des élèves et des établissements d'enseignement a de toute évidence un impact important sur la performance. Les autorités françaises se devaient de réagir quant aux piètres performances du pays, mais la réponse proposée (sans rentrer dans la caricature) ne semble pas vraiment à la hauteur des enjeux.

Que peut-on retenir de l'annonce ministérielle et de ce qui a précédé récemment, c'est-à-dire la conception et la mise en place des nouveaux programmes en seconde en 2010, puis en première à la rentrée 2011 ?

L'Enseignement Intégré de Science et Technologie sera étendu aux collèges "Réseau Ambition Réussite ". Rappelons qu'il s'agit de faire interagir trois disciplines que sont les Sciences de la Vie et de la Terre, la Physique-Chimie et la Technologie, avec un enseignement scientifique qui vise à promouvoir une pédagogie d'investigation. On ne peut que se réjouir de l'extension de ce dispositif en tout point remarquable. Ensuite, il est annoncé qu'un inspecteur sera nommé dans chaque académie pour mieux piloter le système, renforcer les partenariats entre le ministère et les institutions. Il faut en prendre acte, même si l'efficacité d'une telle mesure ne saute pas aux yeux.

Ce qui est très clair en revanche est l'impact qu'aura, à brève échéance, la diminution d'un tiers du nombre d'heures de sciences au niveau de la première $S$, avec en corollaire l'inévitable report des apprentissages structurants au niveau de la terminale et le manque de temps indispensable pour asseoir le goût pour les sciences, ce dont se targue justement le plan ministériel. Il est à craindre, en particulier, que le constat de désaffection des sciences par les jeunes filles non seulement ne s'améliore pas, mais s'aggrave, car ces dernières ont davantage besoin d'être rassurées que les garçons pour entreprendre une formation ou s'engager dans un métier scientifique.

Toute aussi inquiétante pour une discipline éminemment expérimentale comme la nôtre est la perte du caractère obligatoire des travaux pratiques, avec un risque de hiérarchisation des lycées en raison de l'utilisation disparate et variable des heures d'accompagnement personnalisé. On est certes dans le cadre d'une autonomie au sens de PISA, mais il n'est pas certain que le nivellement puisse se faire par le haut faute de moyens suffisants, sans parler des tensions au sein des équipes pédagogiques qui ne manqueront pas dans un même établissement, pour savoir qui obtient quoi et pour qui.

Une autre question importante va concerner très rapidement les formateurs de nos jeunes que sont les professeurs certifiés ou agrégés ; on assiste cette année à un écroulement du nombre d'étudiants en master enseignement. Une analyse et un premier bilan du processus de "mastérisation » sont prévus lors de la session parallèle «Enseignement » du congrès général de la SFP à Bordeaux en juillet 2011. Ceci donnera lieu à une communication spécifique dans un prochain numéro de Reflets de la physique.

Martial Ducloy et Arnaud Le Padellec 